\title{
Intrauterine Repair of Gastroschisis in Fetal Rabbits
}

\author{
H. Tilla ${ }^{a}$ O. Muensterer ${ }^{a} \quad$ M. Mueller ${ }^{a} \quad$ V. Klis ${ }^{a} \quad$ S. Klotz ${ }^{b} \quad$ R. Metzger ${ }^{a}$ \\ I. Joppich ${ }^{a}$ \\ Departments of a Pediatric Surgery, and ' $V$ eterinary Medicine, University of Munich, Munich, Germany
}

\author{
Key Words \\ Gastroschisis · Repair · Closure · Intrauterine $\cdot$ Fetal \\ surgery
}

\begin{abstract}
Objective: Infants with gastroschisis (GS) still face severe morbidity. Prenatal closure may prevent gastrointestinal organ damage, but intrauterine GS repair (GSR) has not been established yet. Methods: In New Zealand White rabbits we developed and compared GS versus GSR: creation of GS was achieved by hysterotomy, right-sided laparotomy of the fetus and pressure on the abdominal wall to provoke evisceration. GSR was accomplished by careful reposition of eviscerated organs and a running suture of the fetal abdominal wall. For study purposes, 18 animals were divided equally into 3 groups: GS, GS with GSR after $2 \mathrm{~h}$, and unmanipulated controls (C). Vitality was assessed by echocardiography. After $5 \mathrm{~h}$ all animals were sacrificed. Results: GSR inflicted no increased mortality, because all fetuses survived GS or GS with GSR. All fetuses with GS demonstrated significant evisceration of abdominal organs. In contrast, the abdominal wall of the fetuses from GSR was intact. Conclusion: The present animal model demonstrated the technical feasibility and success of an intrauterine repair of GS for the first time. However, further long-term studies (leaving
\end{abstract}

\begin{tabular}{ll}
\hline KARGER & ( 2003 S. Karger AG, Basel \\
1015-3837/03/0185-0297\$19.50/0 \\
$\begin{array}{l}\text { Fax +4161306 12 34 } \\
\begin{array}{l}\text { E-Mail karger@karger.ch } \\
\text { www.karger.com }\end{array}\end{array}$ & $\begin{array}{l}\text { Accessible online at: } \\
\text { www.karger.com/fdt }\end{array}$
\end{tabular}

GS and GSR in utero for several days) will be necessary to compare survival rates and intestinal injury, motility or absorption. The clinical application of GSR in utero remains a vision so far.

Copyright $\odot 2003$ S. Karger AG, Basel

\section{Introduction}

Despite immediate surgical repair and qualified intensive care, infants with gastroschisis (GS) face severe morbidity and mortality in up to $79 \%$ of all cases. About half of these complications derive from injury to the gastrointestinal organs, which have been exposed to the amniotic fluid in utero [1, 2]. Postpartum, the intestine of such patients appears dilated or inflamed and reveals dysmotility or malabsorption [3]. Many of these infants require prolonged intensive care with parenteral feeding, multiple reoperations and treatment for the sequelae of intestinal atresia [4]. Based on the clinical situation, a prenatal prevention of fetal organ damage seems a logical approach and justifies the experimental concept of an intrauterine GS repair (GSR). However, so far this procedure has not been established and there are no corresponding animal models. We developed a technique for GSR in fetal New Zealand White rabbits and thereby evaluate the technical feasibility and surgical success of this method.
Holger Till, MD

Childrens' Hospital, University of Munich, Lindwurmstrasse 4

DE-80337 Munich (Germany)

Tel +498951602811, Fax +498951604726

E-Mail holger.till@kk-i.med.uni-muenchen.de 


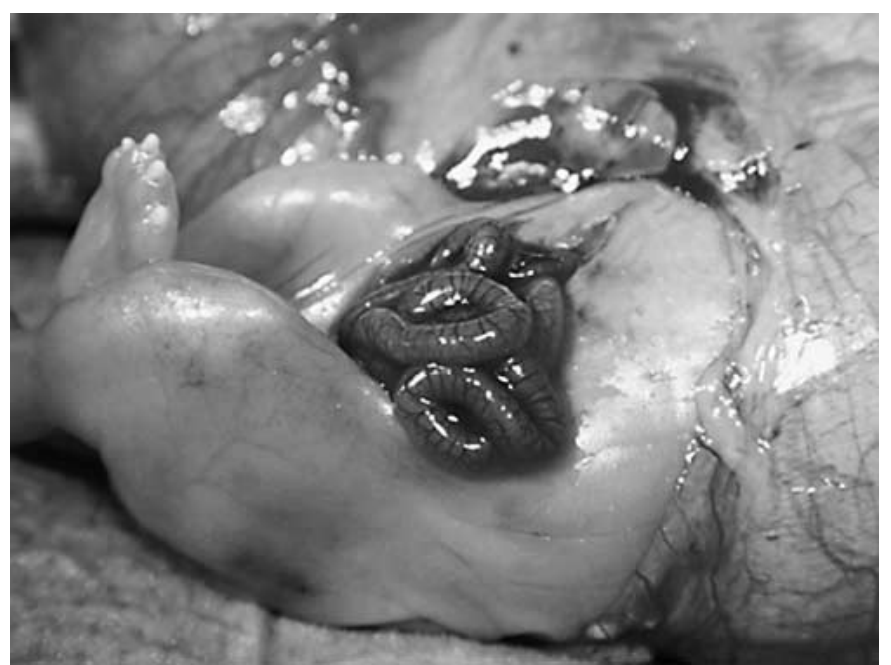

Fig. 1. GS was created by a paraumbilical incision in the right lower quadrant of the fetal abdomen. Evisceration of organs was achieved by gentle pressure on the fetal abdominal wall.

\section{Methods}

For the study, 18 fetal New Zealand white rabbits were randomized equally into one of the following groups: (1) group GS underwent only creation of GS; (2) group GSR underwent GS and repair $2 \mathrm{~h}$ later; (3) group C consisted of unmanipulated fetuses as controls. All fetuses were monitored by echocardiography for a total period of $5 \mathrm{~h}$, after which they were sacrificed for macroscopic and histological evaluation.

For creation of GS we adopted the experimental model of Phillips [5]: On day 27 of gestation (term 31 days), 6 pregnant New Zealand White rabbits with a mean weight of $3.8 \mathrm{~kg}$ underwent median laparotomy to identify the bicornuate uterus. After premedication with a subcutaneous dose of $0.5 \mathrm{mg}$ atropine for vagolysis and $10 \mathrm{mg}$ of diazepam, anesthesia was induced using $40 \mathrm{mg}$ midazolam s.c., $0.4 \mathrm{mg}$ medetomidin i.m., and $40 \mathrm{mg}$ ketamine i.m. As soon as the corneal reflexes were absent, intubation was performed with a straight blade laryngoscope and a $3.5-\mathrm{mm}$ endotracheal tube. The mother rabbit was monitored by EKG tracing, pulse oxymetry recorded from the left upper extremity, and a rectal temperature probe. Body temperature was maintained between 37.0 and $38.0^{\circ} \mathrm{C}$ by placing the animal on a warming blanket. Anesthesia was maintained during the experiment by continuous infusion of $80 \mu \mathrm{g} / \mathrm{kg} / \mathrm{min}$ of ketamine and propofol, titrated for adequate sedation. Tocolysis was achieved by continuous intravenous administration of terbutalin $2 \mu \mathrm{g} / \mathrm{kg} / \mathrm{min}$ and atosiban $8 \mu \mathrm{g} / \mathrm{kg} / \mathrm{min}$. The fetuses were assessed for their intrauterine position and vitality by ultrasound (defining a heart rate of more than 100 beats per minute (bpm) as vital). Above the fetal abdomen, a 1-cm-long hysterotomy was performed on the antimesometrial border of the uterus through the myometrium, the chorionic and amniotic membranes, thereby opening the amniotic cavity. GS was created by a $5-\mathrm{mm}$ longitudinal paraumbilical incision in the left lower quadrant of the abdomen. Evisceration of organs was achieved by gentle pressure on the fetal abdominal wall

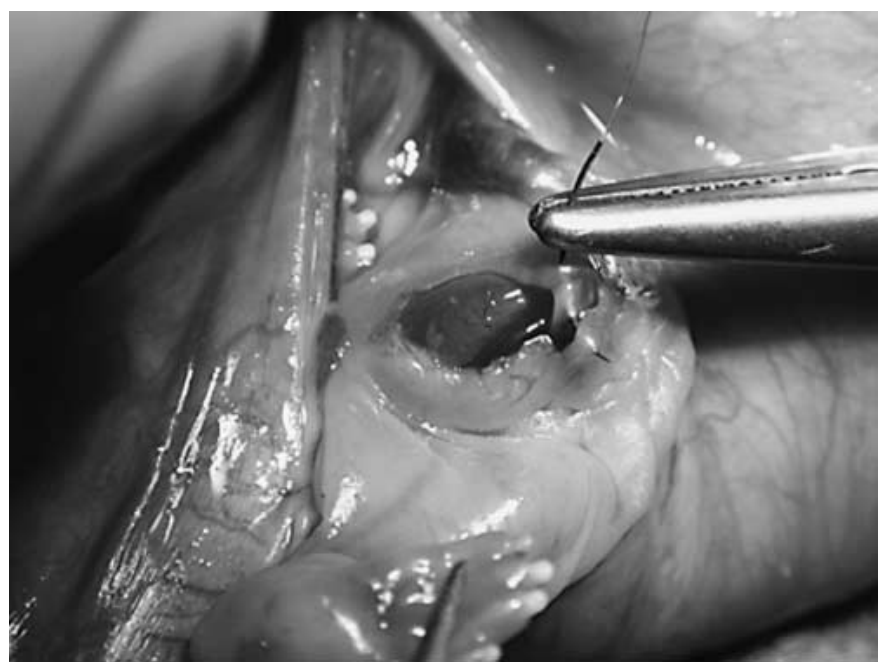

Fig. 2. After reposition of eviscerated organs, the fetal abdomen was closed (GSR) with a running suture (prolene 7-0), avoiding the umbilical vessels at the inside of the fetal abdominal wall.

(fig. 1). Thereafter, each fetus was repositioned and the hysterotomy was closed with a 5-0 polypropylene running suture (Ethicon, Eppendorf, Germany) after reinstallation of the amniotic fluid, taking care to identify and include the amniotic, the chorionic membrane and the uterine musculature. To achieve GSR, the hysterotomy was reopened by removing the running suture. Each fetus was carefully turned to expose the GS. The abdominal wall was then grasped close to the insertion of the umbilical cord and the eviscerated organs were gently pushed back into the fetal abdominal cavity with a moist cotton tipped swab, permitting closure of the abdominal wall with a 7-0 polypropylene running suture while taking special care to avoid the umbilical vessels (fig. 2). Finally, the hysterotomy was sutured in the fashion described above.

\section{Results}

According to intermittent echocardiographic monitoring, all fetuses in the GS and GSR group survived the study period, while there were 2 deaths in the control group, one due to preterm labor. Figure 3 demonstrates the heart rates of each animal for GS, GSR and C: Within $1 \mathrm{~h}$ after creation of GS, the heart rates dropped slightly and recovered spontaneously thereafter. At $2 \mathrm{~h}$, repair of GSR was performed, and again only a minor postoperative decrease of the mean heart rates was noted. At $5 \mathrm{~h}$ the study was terminated and the fetuses were explanted for macroscopic and histological evaluation. All fetuses with GS showed significant evisceration of abdominal organs. In contrast, the fetuses with GSR demonstrated a closed abdominal wall without evidence of a residual defect or 
Fig. 3. Heart rates of each animal from GS, GSR and C: Within $1 \mathrm{~h}$ after creation of GS the heart rates dropped from a mean of 200 $\mathrm{bpm}$ to around $150 \mathrm{bpm}$, but heart rates recovered thereafter. At $2 \mathrm{~h}$, repair of GSR was performed, but no decrease of heart rates was noted this time.

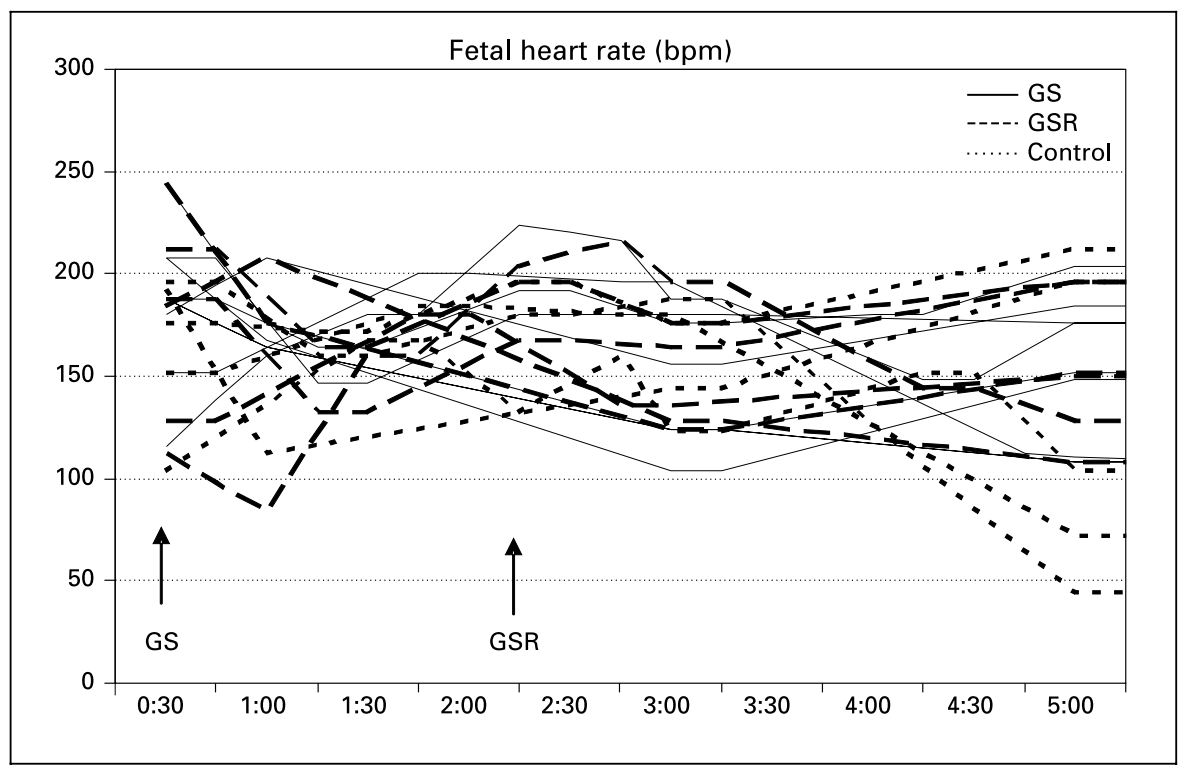

herniation (fig. 4). Histological evaluation of intestinal specimens from GS and GSR found no difference concerning serosal edema or wall thickening.

\section{Discussion}

Infants with GS face a variety of problems in the perinatal period. Surgical closure of the defect is urgent and may be complicated by intestinal wall thickening, insufficient volume of the abdominal cavity and decreased IVC blood flow [6]. In the postoperative course Durfee reported that $79 \%$ of his patients experienced severe complications (e.g. infections, multisystemic failures) and more than one third must be attributed to gastrointestinal pathologies like dysmotility or malabsorption [1]. Fetal protein loss has been postulated as one of the etiologic factors for fetal demise [3]. Most of these infants required prolonged intensive care, parenteral support or secondary surgery and still about $10 \%$ faced death. This significant morbidity and mortality of children with GS raises the question, whether they would benefit from a prenatal closure of the defect. Routine prenatal sonographic screening allows for early detection of these defects [7], providing the basis for prenatal intervention and treatment. Interestingly, a spontaneous intrauterine closure of the defect in humans has been reported a few times $[8,9]$.

To address this problem experimentally, we adopted a well-known animal model for the creation of a GS [5].

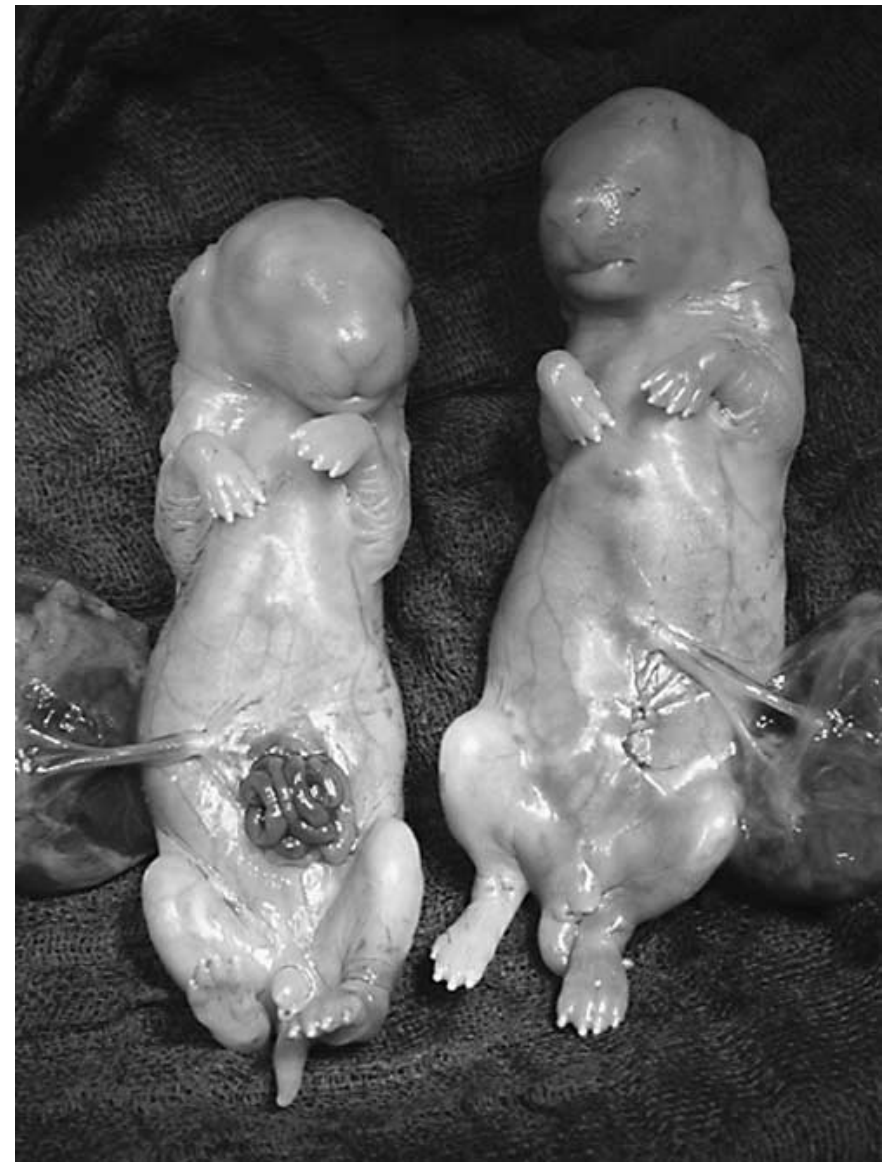

Fig. 4. The fetus following GS reveals significant evisceration of abdominal organs, while the fetus following GS plus GSR shows a patent abdominal wall. 
Phillips has proven earlier that his technique is efficient to cause a significant abdominal wall defect and achieve substantial evisceration of fetal organs. Although gastroschisis usually occurs to the right of the umbilicus due to disruption of the omphalomesenteric artery [10], we chose to perform the defect on the left to avoid injury to the ductus venosus during manipulation in our initial experiment. We have since then successfully created right abdominal defects in an analogous technique without difficulty. Naturally, the experimental incision of the abdominal wall cannot reproduce the embryonic malformation in humans, but his study demonstrated that the model mimics the clinical situation of GS and reproduces the intrauterine damages to the viscera well [5]. Therefore, it seemed appropriate as a model to develop a surgical technique for an intrauterine repair. Since the focus of the present study was to establish a technique for a repair of GS, the authors refrained from a detailed evaluation of the severity of the GS and started GSR rather early. This short interval between creation and repair of GS explains the lack of morphological differences between intestinal specimens from GS versus GSR. Phillips reported that the evisceration of organs and exposure to the amniotic fluid in experimental GS needs to last for several days before tissue edema and injuries were noted. This observation represents the central argument for our study: if an intrauterine repair of GS could be accomplished before major damages to the organs appeared, some of the important clinical problems of GS after birth, such as an incongruence of volumes between the abdominal cavity and the intestine or a compromised venous blood flow, could be avoided. Moreover, if the correction could be performed early enough in utero, enlargement of the fetal abdominal cavi- ty may not be necessary. These speculations must be evaluated in further studies, now that the basic technical details of GSR have been established. The main surgical goal of the present study however could be accomplished with success: After a complete study period of $5 \mathrm{~h}$ all animals of GS and GSR were alive with heart rates above $100 \mathrm{bpm}$. This survival rate not only compares to other investigators, who created a GS in rabbits (e.g. 89\% in Phillips study [5]), but moreover it means that repair of GS inflicted no significant mortality upon the fetuses. Surgical success could be demonstrated in all animals with GS repair, making this technique feasible and safe. However, of 2 deaths in the control group one occurred due to preterm labor. Tocolysis is certainly crucial to fetal surgery, because the uterus reacts extremely sensitively and contracts easily, when being manipulated or sutured [11]. The fact that the overall incidence of preterm labor in the present study was only 1 of 18 animals proves that terbutalin and atosiban were powerful and efficient drugs in this regard.

In conclusion, the present animal model demonstrated for the first time the technical feasibility and success of an intrauterine repair of GS. However, further long-term studies (leaving GS and GSR in utero for several days) will be necessary to compare survival rates, intestinal injury, motility or absorption. Due to the current morbidity and fetal mortality of prenatal intervention, the clinical application of GS repair in utero remains a vision. Once the challenges of preterm labor, secure long-term closure of the uterine membranes, and potential choreoamnionitis are overcome, prenatal closure of isolated abdominal wall defects may represent a valuable alternative to postnatal repair.

\section{References}

1 Durfee SM, Downard CD, Benson CB, Wilson JM: Postnatal outcome of fetuses with the prenatal diagnosis of gastroschisis. J Ultrasound Med 2002;21:269-274.

2 Burge DM, Ade-Ajayi N: Adverse outcome after prenatal diagnosis of gastroschisis: The role of fetal monitoring. J Pediatr Surg 1997;32: 441-444.

3 Carroll SG, Kuo PY, Kyle PM, Soothill PW: Fetal protein loss in gastroschisis as an explanation of associated morbidity. Am J Obstet Gynecol 2001;184:1297-1301.
4 Tunell WP, Puffinbarger NK, Tuggle DW, Taylor DV, Mantor PC: Abdominal wall defects in infants. Survival and implications for adult life. Ann Surg 1995;221:525-528.

5 Phillips JD, Kelly RE, Fonkalsrud EW, Mirzayan A, Kim CS: An improved model of gastroschisis in fetal rabbits. J Pediatr Surg 1991; 26:784-787

6 Srinathan SK, Langer JC, Botney MD, Pelletier GJ: Submucosal collagen in experimental gastroschisis. J Surg Res 1996;65:25-30.

7 Martin RW: Screening for fetal abdominal wall defects. Obstet Gynecol Clin N Am 1998;25: 517-526.
8 Bhatia AM, Musemeche CA, Crino JP: Gastroschisis complicated by midgut atresia and closure of the defect in utero. J Pediatr Surg 1996;31:1288-1289.

9 Tawil A, Comstock $\mathrm{CH}$, Chang $\mathrm{CH}$ : Prenatal closure of abdominal defect in gastroschisis: Case report and review of the literature. Pediatr Dev Pathol 2001;4:580-584.

10 Hoyme HE, Higginbottom MC, Jones KL: The vascular pathogenesis of gastroschisis: Intrauterine interruption of the omphalomesenteric artery. J Pediatr 1981;98:228.

11 Harrison MR: Fetal surgery. Am J Obstet Gynecol 1996;174:1255-1264. 